\title{
Role of the Venereal Disease Research Laboratory test in the detection of syphilis
}

\author{
PAUL DIGGORY \\ From the Department of Community Medicine, University of Southampton, Southampton
}

SUMMARY Of 9733 consecutive serological samples received by Portsmouth and Southampton Public Health Laboratories (PHL) and tested for syphilis, 190 (140 from men and 50 from women) gave positive results. Thirty new cases of syphilis were identified. Most sera were tested initially by both a specific antibody test (the Treponema pallidum haemaglutination (TPHA) test) and a cardiolipin test (the Venereal Disease Research Laboratory (VDRL) test). Among the 14 patients whose sera gave VDRL-positive but TPHA-negative results, 12 sera gave false-positive results. The sera of 90 patients gave TPHA-positive but VDRL-negative results; sera from only seven of these patients gave false-positive results.

The VDRL test is very unlikely to identify a new case of syphilis where a TPHA test has failed to do so. The results of the survey suggest that the VDRL test should be withdrawn from initial testing for syphilis except where early primary disease is suspected.

\section{Introduction}

There are two categories of serological tests for syphilis: cardiolipin tests, which detect antibodies to lipoidal antigens derived from cell membranes; and specific tests, which detect antitreponemal antibody.

The most widely used cardiolipin and specific tests are respectively the Venereal Disease Research Laboratory (VDRL) test ${ }^{1}$ and the Treponema pallidum haemagglutination (TPHA) test. ${ }^{2}$ Both are suitable for large-scale use. The fluorescent treponemal antibody-absorption (FTA-ABS) test is used for confirmation. ${ }^{3}$

Cardiolipin tests are not truly specific for syphilis. ${ }^{45}$ Antibody concentrations may be high in a large number of unrelated diseases, in pregnancy, and as a normal variant in some healthy people. Most febrile illnesses, connective tissue diseases, and chronic infections may be associated with false-positive reactions in these tests. Their value lies in their low cost and in the ease with which they are titred. Specific tests are less likely to give false-positive results in diseases not caused by treponemes, although the tests may give positive results in patients with infectious mononucleosis, lepromatous leprosy, and in a few healthy people. ${ }^{67}$

In primary syphilis the VDRL test is marginally more sensitive than the TPHA. It gives a positive result

\footnotetext{
*This study was undertaken as a student project

Address for reprints: Dr P Diggory, 10 Campden Hill Square, London W8 7LB

Accepted for publication 24 July 1982
}

earlier in the primary stage, soon after the appearance of the chancre, and at a high titre by the secondary stage. Thereafter the titre falls as the disease progresses so that in some $30 \%$ of cases of latent and tertiary syphilis the result is negative.

The TPHA test is more sensitive than the VDRL in all but primary syphilis, and it is the most sensitive test of all for latent disease. In patients who have been treated for syphilis which has progressed past the secondary stage the TPHA result is likely to remain positive for life; this probably reflects the detection by the test of IgG antibody. The TPHA test results on sera from patients treated during the primary and early secondary stages of the disease usually become negative within one to two years. ${ }^{58-11}$

The aims of the present study* were to obtain a profile of serological testing for syphilis, performed by the Southampton and Portsmouth Public Health Laboratories, to relate the initial testing of sera to the ultimate diagnosis, and to evaluate the effectiveness of the tests used.

\section{Materials and methods}

After approval by the relevant ethics committees of a method of preserving patient confidentiality the cardrequests for serological tests for syphilis held by the Portsmouth and Southampton Public Health Laboratories (PHL) were studied. Age, sex, and requesting specialty were noted. For all positive sera the requesting consultant or general practitioner was asked for access to case notes, from which the reason for requesting the test and the final diagnosis were extracted. 
The Portsmouth PHL performed the VDRL and TPHA tests on all serum samples. If the results of either or both tests were positive an FTA-ABS test was carried out. Tests with doubtful results were repeated, and if the diagnosis was still in doubt the serum sample was sent to the Venereal Disease Reference Laboratory at the London Hospital, London.

The VDRL slide flocculation test was performed using the VDRL antigen pack (Wellcome Reagents Ltd, Beckenham, UK). The TPHA test was a modified micro-method as described by Johnston. ${ }^{12}$ The reagents used in the FTA-ABS test were obtained from Wellcome Reagents Ltd; the laboratory used its own syphilitic sera as controls.

In Southampton PHL all sera were initially tested by the TPHA and VDRL tests except those from antenatal and genitourinary medicine clinics; these are tested by the TPHA test alone. When positive results were obtained the sera were examined by the VDRL and FTA-ABS tests. A separate record of all positive serological results was kept and consulted before a FTA-ABS test was performed. Unless a new infection was suspected the FTA-ABS test was not repeated if previously the result had been positive. As in Portsmouth the TPHA test was performed by a modified micro-method. The VDRL test was carried out using the Fisher diagnostic reagin screen test, a macroscopic card test for syphilis (A R Horwell, London). The FTA-ABS test was performed as in Portsmouth except that sorbent (obtained from Difco, Surrey) was used. Again samples of syphilitic serum obtained locally were used as positive controls. For the purposes of the study equivocal results were interpreted as negative.

Southampton PHL performed routine serological testing for both the Southampton and Bournemouth genitourinary medicine clinics.

\section{Results}

The survey ran for six months in Portsmouth and for three months in Southampton. A total of 9733 samples of sera were analysed. Of these, 7187 were initially tested by both the TPHA and VDRL tests and 2546 by the TPHA test alone. Antenatal clinics provided 5140
$(52 \cdot 8 \%)$ sera and genitourinary medicine clinics 2541 $(26 \cdot 1 \%)$ sera.

One hundred and ninety patients (140 male, 50 female) whose serological test results for syphilis were positive were identified (table). One hundred and thirty-three patients (114 male, 19 female) had attended a genitourinary medicine clinic and 17 an antenatal clinic. Most positive sera in men were from patients who had had, or were being treated for, syphilis. Most women with positive test results had biological false-positive results; most of these women had attended an antenatal clinic. Nineteen sera showed biological false-positive results; there were five patients with yaws and a positive test result was obtained in a neonate as a result of passive transfer of antibody. In all, 30 new cases of syphilis were found; 25 of these patients were men.

\section{VDRL-POSITIVE/TPHA-NEGATIVE RESULTS}

Fourteen patients had VDRL-positive but TPHAnegative results; 12 of these were false-positive results with no evidence of treponemal infection. One patient gave a history of syphilis treated $\mathbf{3 0}$ years previously and one had primary syphilis. In all cases, apart from the patient with primary syphilis, the FTA-ABS test result was negative.

TPHA-POSITIVE/VDRL-NEGATIVE RESULTS Ninety patients had TPHA-positive but VDRLnegative results; 62 had been treated previously for syphilis and one had had yaws. Sera from 10 patients were tested during the follow-up of the treatment of the disease. Two patients were found to have yaws. The serum from a newborn child gave a positive test result due to passive transfer of antibody from his mother. There were seven biological false-positive results; the FTA-ABS test result was negative in each case, and seven new cases of syphilis were identified.

\section{Discussion}

In view of the low incidence of syphilis today ${ }^{13}$ and the importance of reducing costs the number of tests for syphilis needs to be kept to a minimum. Except in cases of primary disease the VDRL test is very unlikely to identify syphilis where the TPHA has failed to do so. Moreover, it produces more false-positive results at all

TABLE FTA-ABS test results on the sera of patients whose TPHA or VDRL tests or both gave positive results on initial testing

\begin{tabular}{|c|c|c|c|c|}
\hline \multirow[b]{2}{*}{ Initial testing } & \multicolumn{4}{|c|}{ No of sera with following serological test results: } \\
\hline & FTA-positive & FTA-negative & FTA (not done) & Total \\
\hline $\begin{array}{l}\text { VDRL-positive/TPHA-positive } \\
\text { VDRL-negative/TPHA-positive } \\
\text { VDRL-positive/TPHA-negative }\end{array}$ & $\begin{array}{r}66 \\
62 \\
1\end{array}$ & $\begin{array}{r}1 \\
16 \\
13\end{array}$ & $\begin{array}{r}19 \\
12 \\
0\end{array}$ & $\begin{array}{l}86 \\
90 \\
14\end{array}$ \\
\hline Total & 129 & 30 & 31 & 190 \\
\hline
\end{tabular}


stages and more false-negative results in late disease. Although most infectious syphilis is homosexually transmitted ${ }^{14}$ and treated at genitourinary medicine clinics most requests come from other specialties where primary syphilis is not generally suspected. Despite the lower costs of the VDRL test (in terms of reagents, about $10 \%$ of those of the TPHA) if it only became available today it would very probably not be introduced at all.

A TPHA-positive/VDRL-negative result on a patient's serum implies that he has or has had a treponemal infection. In this survey of the 90 patients whose sera were TPHA-positive but VDRL-negative only seven had false-positive results, and seven new cases of syphilis were identified.

If the TPHA test result is negative the patient is unlikely to have syphilis even if the VDRL test result is positive. This view is supported by the data from the Southampton and Portsmouth laboratories. Of the 14 patients whose sera were VDRL-positive but TPHAnegative, 12 had no treponemal infection. One gave a history of treated syphilis (though as no specific test gave a positive result the patient may well never have had the disease). The one new case identified was a patient who attended a genitourinary medicine clinic. He had gonorrhoea but also unsuspected early primary syphilis. A FTA-ABS test result was positive but the TPHA result was equivocal. The man returned for follow-up and repeat tests all gave positive results.

Patients whose sera give positive results to both the TPHA and VDRL tests are very likely to have been infected by treponemes at some time in their life. In this survey one patient whose serum was VDRLpositive and TPHA-positive but FTA-ABS-negative was thought to have been treated previously for syphilis.

Genitourinary medicine clinics accounted for $26 \%$ of the requests in the survey and are the most likely to see patients with primary disease. The staff at the clinics have great experience in interpreting serological tests for syphilis and are unlikely to be confused by biological false-positive VDRL results. Consequently such clinics are the most likely to benefit from initial testing of sera by the VDRL and TPHA tests.

Genitourinary medicine clinics apart, syphilis serology is almost always requested to exclude disease beyond the primary stage. The TPHA test result will be positive, and a VDRL test adds little to the identification of the disease. Many doctors, unfamiliar with the modern tests, may be misled by a VDRL-positive/TPHA-negative result into thinking that the patient has syphilis.

Antenatal clinics perform routine serological tests for syphilis as a screening measure. Although the occurrence of primary disease is possible it will almost certainly have passed this stage by the time of booking.
If primary syphilis is to be ruled out then testing must logically be repeated at every visit as the woman may contract the disease during pregnancy. It is worth remembering that pregnancy is a recognised cause of a false-positive VDRL test result.

At Southampton the load on the PHL has been reduced by testing sera from genitourinary medicine and antenatal clinics initially by the TPHA test alone. The results of this survey suggest that this is justified for antenatal clinic patients but that there is a theoretical risk of missing cases of primary syphilis at genitourinary medicine clinics. In other specialties initial testing by the TPHA test alone is adequate except where primary disease is suspected clinically.

The TPHA test result can be titred but the reagents are expensive and the titres high. The VDRL test provides an excellent, inexpensive method of assessing disease activity and monitoring treatment. It gives low titres or becomes negative with inactive or treated disease and gives high titres in active syphilis. In view of the need to reduce costs a policy of initial testing by the TPHA test alone with the VDRL test for titration and an FTA-ABS test for confirmation should be considered. Because syphilis is such a serious, but treatable disease initial testing by the VDRL test alone without a specific test is not justified, as a proportion of latent and tertiary disease would be missed.

I wish to acknowledge the help of Drs $\mathbf{M} \mathrm{J}$ Balsdon, SP Donnan, and A D Pearson, Mr B Parsons, and the late Dr D J H Payne.

References

1. Harris A, Rosenberry AA, Riedel LM. A microflocculation test for syphilis using cardiolipin as antigen. J Ven Dis Inf 1946; 27:169-74.

2. Tomizawa T, Kasamaton S, Yamata S. The usefulness of the haemaglutination test using $T$ pallidum as antigen for serodiagnosis of syphilis. Jpn J Med Sci Biol 1969;22:341-5.

3. Hunter EF, Deacon WE, Meyer PC. An improved FTA test for syphilis. The absorption procedure (FTA-ABS). Public Health Rep 1964;79:410-12.

4. Moore JE, Mohr CF. Biological false-positive serological tests for syphilis. JAMA 1952;150: 467-73.

5. Long R, Haserick JR. Systemic lupus erythematosus preceded by false-positive tests for syphilis. Ann Int Med 1952;37:559-62.

6. Mackay M, Price EV, Knox JM, Scotti A. Specificity of the FTA ABS tests for syphilis. JAMA 1969;207:1683-5.

7. O'Neill P, Warner RW, Nicol CS. TPHA assay in the routine serodiagnosis of syphilis. Br J Vener Dis 1973;49:427-31.

8. Deacon WE, Lucas JE, Price EV. Fluorescent treponemal antibody tests for syphilis. JAMA 1966;198:624-28.

9. Young H, Henrichsen C, Robertson DHH. The TPHA test as a screening procedure for the diagnosis of syphilis. Br J Vener Dis 1974;50:341-6.

10. Lesinski J, Krach J, Kadzienicz E. Specificity, sensitivity and diagnostic value of the TPHA test. Br J Vener Dis 1974;50:334-40.

11. Fiumara NJ. Treatment of secondary syphilis: an evaluation of 204 patients. Sex Transm Dis 1977;4:96-9

12. Johnson NA. The TPHA test for syphilis. Evaluation of a modified micro-method. Br J Vener Dis 1972;48:474-7.

13. Anonymous. Sexually transmitted disease surveillance. Br Med J 1979;i:155.

14. Fluker JL. Homosexuality and sexually transmitted diseases. $\mathrm{Br}$ J Hosp Med 1981;26:265-8. 